Article

\title{
Synthesis of Polyamidoamine Dendrimer for Encapsulating Tetramethylscutellarein for Potential Bioactivity Enhancement
}

\author{
Daniel M. Shadrack ${ }^{1,2}$, Egid B. Mubofu ${ }^{1, *}$ and Stephen S. Nyandoro ${ }^{1, *}$ \\ Received: 13 June 2015 ; Accepted: 8 October 2015 ; Published: 4 November 2015 \\ Academic Editor: Graeme Cooke \\ 1 Chemistry Department, University of Dar es Salaam, College of Natural and Applied Sciences, \\ P.O. Box 35061 Dar es Salaam, Tanzania; dmssjut@gmail.com \\ 2 Chemistry Department, St John's University of Tanzania, P.O. Box 47 Dodoma, Tanzania \\ * Correspondence: ebmubofu@udsm.ac.tz (E.B.M.); nyandoro@udsm.ac.tz (S.S.N.); \\ Tel.: +255-784-538-344 (E.B.M.); +255-754-206-560 (S.S.N.); Fax: +255-22-410-038 (E.B.M. \& S.S.N.)
}

\begin{abstract}
The biomedical potential of flavonoids is normally restricted by their low water solubility. However, little has been reported on their encapsulation into polyamidoamine (PAMAM) dendrimers to improve their biomedical applications. Generation four (G4) PAMAM dendrimer containing ethylenediaminetetraacetic acid core with acrylic acid and ethylenediamine as repeating units was synthesized by divergent approach and used to encapsulate a flavonoid tetramethylscutellarein (TMScu, 1) to study its solubility and in vitro release for potential bioactivity enhancement. The as-synthesized dendrimer and the dendrimer-TMScu complex were characterized by spectroscopic and spectrometric techniques. The encapsulation of $\mathbf{1}$ into dendrimer was achieved by a co-precipitation method with the encapsulation efficiency of $77.8 \% \pm 0.69 \%$ and a loading capacity of $6.2 \% \pm 0.06 \%$. A phase solubility diagram indicated an increased water solubility of $\mathbf{1}$ as a function of dendrimer concentration at $\mathrm{pH} 4.0$ and 7.2. In vitro release of $\mathbf{1}$ from its dendrimer complex indicated high percentage release at $\mathrm{pH}$ 4.0. The stability study of the TMScu-dendrimer at 0,27 and $40{ }^{\circ} \mathrm{C}$ showed the formulations to be stable when stored in cool and dark conditions compared to those stored in light and warmer temperatures. Overall, PAMAM dendrimer-G4 is capable of encapsulating 1, increasing its solubility and thus could enhance its bioactivity.
\end{abstract}

Keywords: PAMAM G4 dendrimer; encapsulation; tetramethylscutellarein; solubilization; in vitro release; stability

\section{Introduction}

The development of biodegradable polymeric nanoparticles for effective drug and gene delivery has significantly increased in recent years [1]. Of these, polyamidoamine (PAMAM) dendrimers have been of significant interest due to their unique properties, which provide an endless application in drug delivery. For instance, they are used for gene therapy, drug delivery and magnetic resonance imaging [2]. The use of dendrimers in drug delivery or carrier system to increase therapeutic index, efficacies and solubility in aqueous media is well reported [3,4].

One of the intrinsic properties that limit the clinical applications of the potentially active natural products and their synthetic analogues is poor water solubility [5]. A number of techniques have been used to increase solubility of sparingly water-soluble compounds, among them, being the drug-dendrimer complexation [2]. The ability of PAMAM dendrimers to encapsulate and complex with drugs and other biologically active natural products is based on their unique properties such 
as monodispersity, large surface area, high degree of molecular uniformity, specific size and shape which are significantly different from their classical linear polymer counterparts [6]. Thus, the drug-dendrimer complexation is currently widely used in pharmaceutical industries as an approach to increase stability, solubility, bioavailability and controlled release of drugs [2,6].

Flavonoids and other polyphenolic compounds are known to possess cytotoxic activities on different cancer cells and have other biomedical potentials $[7,8]$. However, water-insolubility and slow dissolution rate remains a serious drawback for their clinical applications [7,9]. Despite the plethora of evidence on PAMAM dendrimers to mediate medical application of most natural products, little have been reported on the encapsulation of flavonoids onto PAMAM and other encapsulating agents [9-11]. Thus, the essence of undertaking investigations involving PAMAM and a flavonoid 5,6,7-trimethoxy-2-(4-methoxyphenyl)-4H-chromen-4-one (Tetramethylscutellarein (TMScu, 1), (Figure 1)). The compound is known to exhibit anti-inflammatory properties [12] and moderate cytotoxic activity against human breast cancer cell lines (MCF-7) and anti-tuberculosis potency against Mycobacterium tuberculosis (H37Rv strain) [13], whereas its structural analogues are well acknowledged for treatment of various ailments $[8,14]$. Dendrimers effectiveness for encapsulation, solubolization and drug delivery depends on the number of generations and terminal group among other factors [3-6]. Hence, our investigations explored the capacity of the amine terminated PAMAM G4 dendrimer to encapsulate compound 1, a model compound among other bioactive flavonoids [13] for potential bioactivity enhancement. The compound solubilization under the influence of the synthesized G4 PAMAM dendrimer, in vitro release and stability in the dendrimer complex were studied, the results of which are reported in this paper.

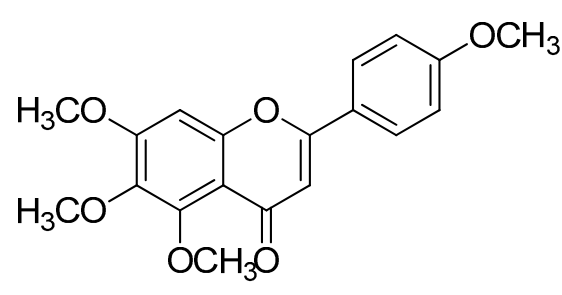

1

Figure 1. Chemical structure of tetramethylscutellarein (TMScu, 1).

\section{Results and Discussion}

\subsection{Synthesis and Characterization of Polyamidoamine (PAMAM) Generation Four (G4) Dendrimer}

The synthesis of PAMAM G4 dendrimer was carried out by a divergent approach with reagent excess, wherein ethylenediaminetetraacetic acid (EDTA) was used as core. All synthetic steps were completed and confirmed by copper sulfate color chelation reactions. While half generations gave deep blue color, full generations had purple coloration. The purple color formed was due to the reaction of terminal amine groups present in the dendrimer with copper sulfate, in half generation, the blue color of copper sulfate was observed signifying the absence of terminal amine groups. The Ultraviolet-visible (UV-Vis) spectrometry further provided the proof of synthesis by showing changes in the wavelength values of the dendrimers. The absorption wavelength of the synthesized dendrimer changed from $\lambda_{\max } 310$ to $299,291,287,279,264$, and $250 \mathrm{~nm}$ for G1, G1.5, G2, G2.5, G3, G3.5 and G4, respectively. The change in wavelength was due to the changes in the structure of the dendrimer generations. While the PAMAM dendrimer generation increased, the absorption shifted from high to lower wave numbers.

The Infrared (IR) spectrum of a solid EDTA, a synthetic starting material was acquired parallel with that of the synthesized PAMAM G4 dendrimer for comparison purpose (Figure 2). The IR spectrum of the EDTA showed absorption due to the presence of carboxylic carbon at $1680.9 \mathrm{~cm}^{-1}$. 
The IR spectrum of the synthesized dendrimer G4 showed absorption peaks at $3353.4 \mathrm{~cm}^{-1}$ for N-H stretching of primary amine, 3277.4 and $3182.4 \mathrm{~cm}^{-1}$ for $\mathrm{N}-\mathrm{H}$ stretching of secondary amine. The peaks at $2927.9 \mathrm{~cm}^{-1}$ and $2856.5 \mathrm{~cm}^{-1}$ were for aliphatic $\mathrm{C}-\mathrm{H}$ stretches. A peak at $1654.5 \mathrm{~cm}^{-1}$ was assigned for amide carbonyl absorption while a peak at $1568.4 \mathrm{~cm}^{-1}$ was due to a core N-C stretching. A disappearance of the IR absorption band at $1680.9 \mathrm{~cm}^{-1}$ for carboxylic carbon for EDTA and appearance of IR absorption at $1654.5 \mathrm{~cm}^{-1}$ for the PAMAM G4 dendrimer was a further evidence of the success of the synthetic transformation carried out.

The Matrix-Assisted Laser Desorption/Ionization Time of Flight Mass Spectrum (MALDI-TOF MS) shown in Figure 3, does not show appreciable peaks at the region expected for molecular ion corresponding to theoretical Mol. Wt. $(14.21 \mathrm{kDa})$, instead it contains a broad peak at a region ideal for half molecular mass for PAMAM G4 (approximately $7.10 \mathrm{kDa}$ ) corresponding to $[\mathrm{M}]^{2+}$. Similar phenomenon including molecular mass deviations and dendrimer structural defects under MALDI MS determination has been reported $[15,16]$. Missing molecular ion and peak broadening at half-size for the desired molecular mass is ascertained to random fragmentations under applied MALDI operating energy, the latter observation coinciding with $[\mathrm{M}]^{2+}$ indicates possible presence of as-synthesized PAMAM G4.

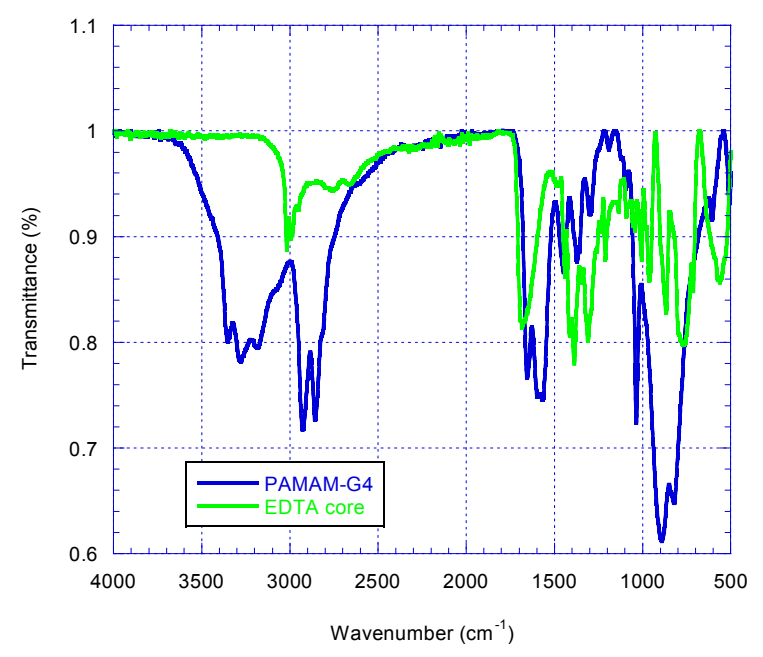

Figure 2. The superimposed IR spectra of solid EDTA (green) and PAMAMG4 (blue).

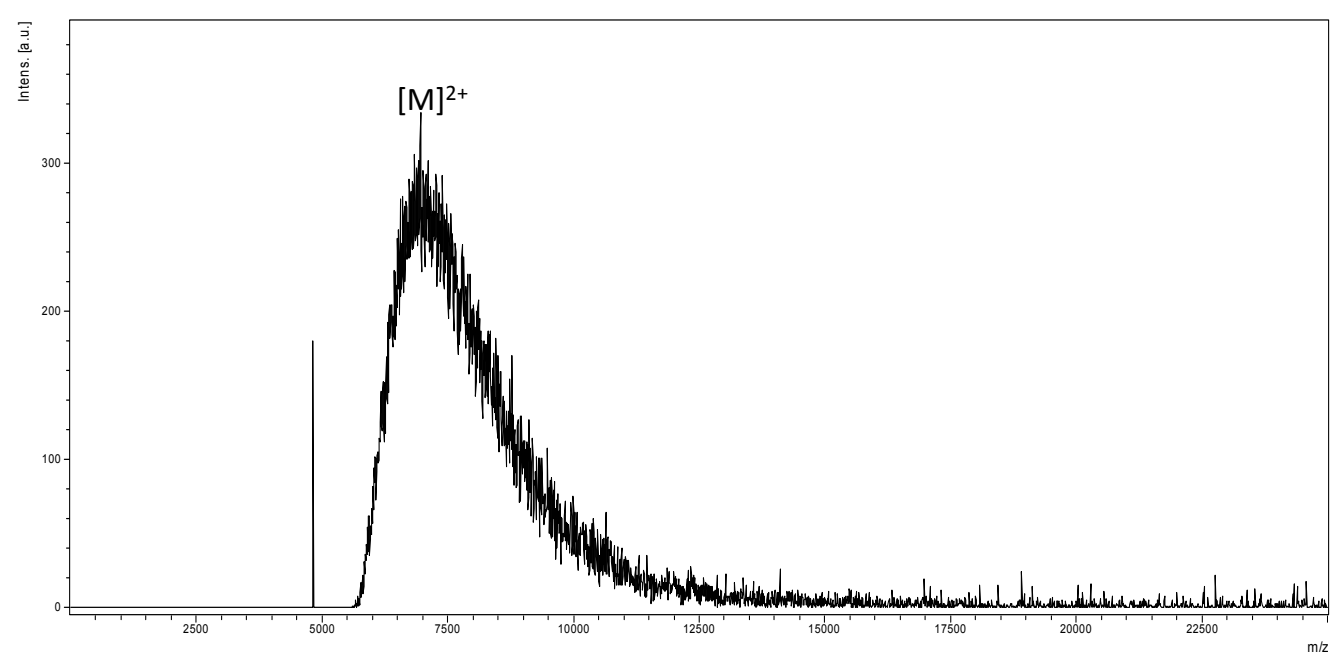

Figure 3. MALDI-TOF mass spectrum of PAMAM G4 acquired using Trans-2-(3-(4-tertbutylphenyl)-2-methyl-2-propenylidene)malononitrile (DCTB) as a matrix. 
The proton nuclear magnetic resonance $\left({ }^{1} \mathrm{H}\right.$ NMR) spectrum of PAMAM G4 dendrimer (Figure 4) consisted of set of signals due to only six different protons correlated to partial structure in Figure 5. The proton peaks were designated as $\mathrm{H}_{\mathrm{a}}, \mathrm{H}_{\mathrm{b}}, \mathrm{H}_{\mathrm{c}}, \mathrm{H}_{\mathrm{d}}, \mathrm{H}_{\mathrm{e}}$ and $\mathrm{H}_{\mathrm{f}}$. The most deshielded peak resonating at $\delta 4.02$ was assigned to amide protons $\left(\mathrm{H}_{\mathrm{a}}\right)$ while the proton signal resonating at $\delta 3.48$ was assigned to terminal amine protons $\left(\mathrm{H}_{\mathrm{b}}\right)$. The proton peak resonating at $\delta 1.93$ was assigned to methylene protons near the carbonyl group $\left(\mathrm{H}_{\mathrm{c}}\right)$. Furthermore, the peak at $\delta 1.72$ was assigned to methylene protons next to the amido nitrogen $\left(\mathrm{H}_{\mathrm{d}}\right)$. An upfield peak resonating at $\delta 1.69$ was assigned to methylene protons $\left(\mathrm{H}_{\mathrm{e}}\right)$ adjacent to the terminal amine. The most shielded upfield peak resonating at $\delta 1.35$ was assigned to methylene protons inside the core $\left(\mathrm{H}_{\mathrm{f}}\right)$ and those near tertiary amine $\left(\mathrm{H}_{\mathrm{f}^{\prime}}\right)$.

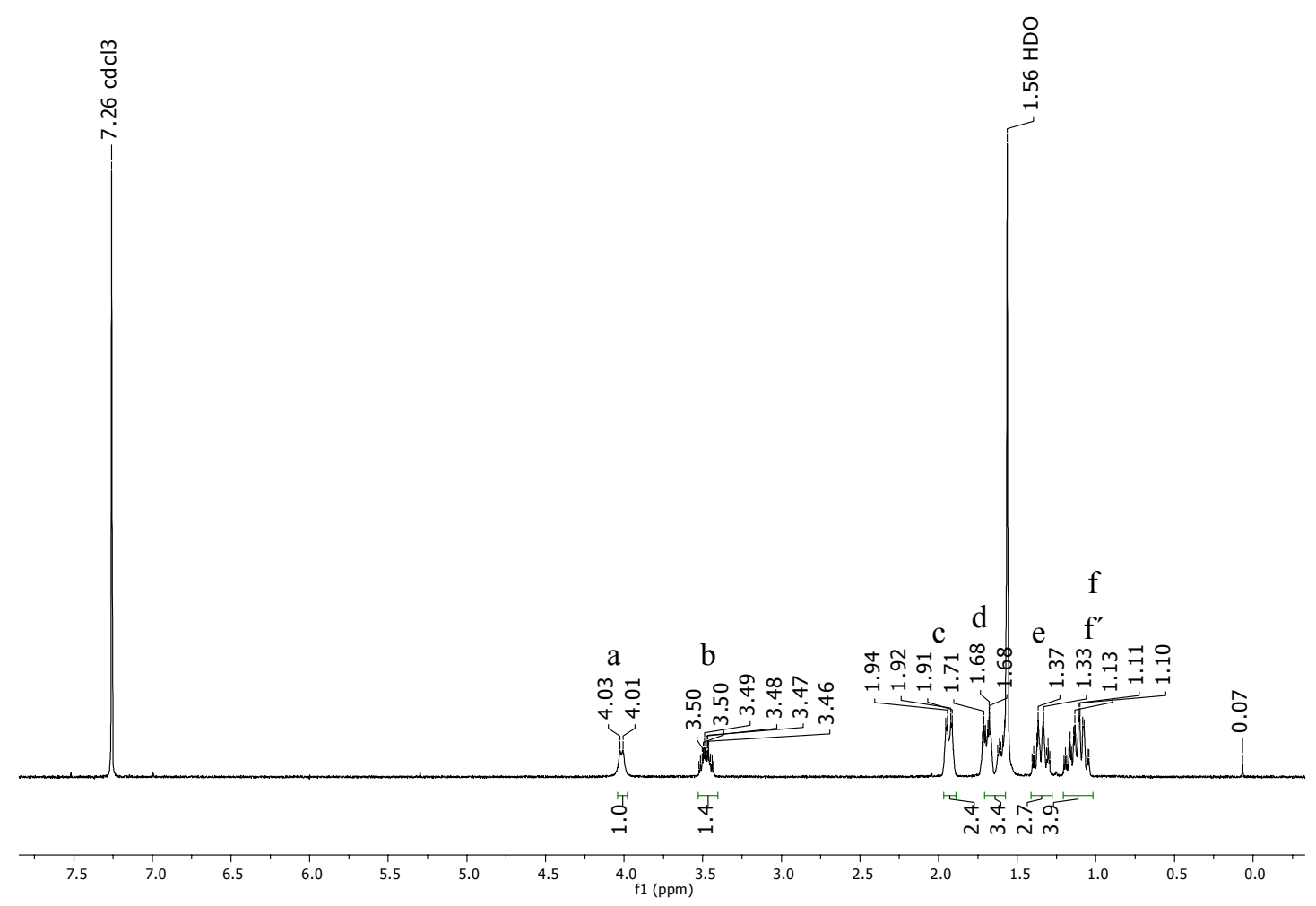

Figure 4. The ${ }^{1} \mathrm{H}$ NMR spectrum of PAMAM dendrimer $\mathrm{G} 4$ observed at $400 \mathrm{MHz}$ for $\mathrm{CDCl}_{3}$ solution at $25^{\circ} \mathrm{C}$.

The structural analysis of synthesized dendrimer G4 as represented by atom labeled partial structure (Figure 5) is in agreement with the ${ }^{1} \mathrm{H}$ NMR spectrum (Figure 4). The structure consists of six different types of protons. These are amido protons (a), protons at the terminal amino group (b), methylene protons adjacent to carbonyl (c), methylene protons adjacent the amido nitrogen (d), methylene protons next to terminal amino group (e), methylene protons near the tertiary amine $\left(\mathrm{f}^{\prime}\right)$ and the methylene protons of the ethylenediaminetetraacetic acid core (f). Generally, results from the UV-Vis, IR, NMR and MALDI-TOF-MS corroborated those reported in previous studies [15-20]. Therefore, with these evidences, EDTA core PAMAM G4 dendrimer was successfully synthesized and characterized. 


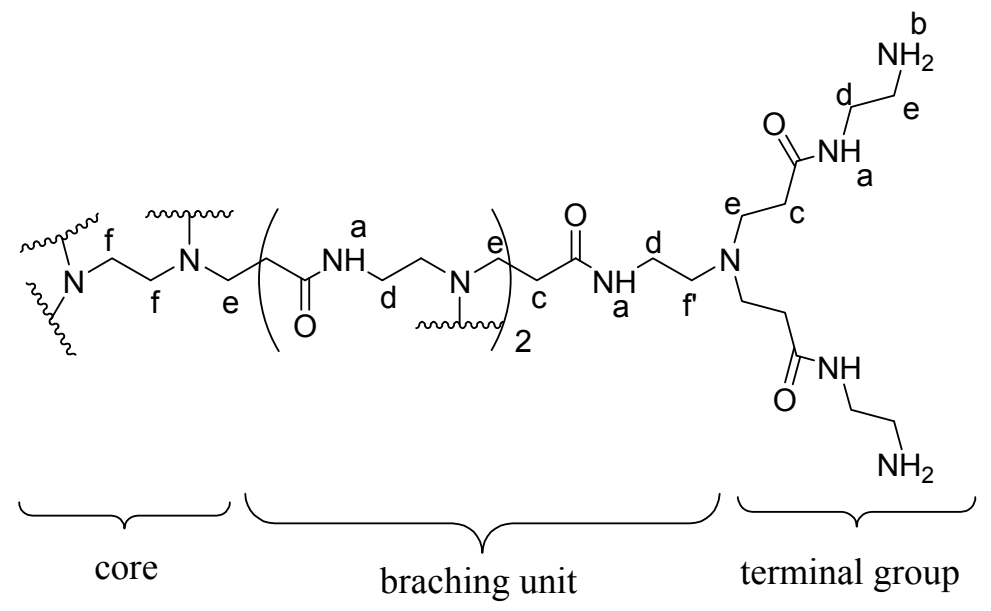

Figure 5. Chemical structure and atom labeling of the part of dendrimer G4.

\subsection{Encapsulation and Characterization of Tetramethylscutellarein (TMScu (1))-Dendrimer Complex}

The encapsulation ability of amine terminated PAMAM G4 dendrimer by complexing with tetramethylscutellarein (TMScu, 1) was investigated using IR, NMR spectroscopic and in vitro release studies. The superimposed IR spectra of TMScu (1) and the compound-dendrimer complex are shown in Figure 6. Compound 1 exhibited IR absorption peak at $1626.4 \mathrm{~cm}^{-1}$ corresponding to aromatic conjugated carbonyl group. The absorption peak at $3075.1 \mathrm{~cm}^{-1}$ was assigned to aromatic ring $\mathrm{C}-\mathrm{H}$ stretch. In PAMAM G4-1 complex, a new broad absorption peak appeared between 3500-3100 $\mathrm{cm}^{-1}$, which indicated the interaction between the dendrimer and the encapsulated compound. The formation and existence of a broad peak (3500-3100 $\left.\mathrm{cm}^{-1}\right)$ with a loop in the region around $3324.1 \mathrm{~cm}^{-1}$ in the IR spectra of PAMAM G4-1 complex suggests the presence of $-\mathrm{NH}_{3}{ }^{+}$ functionality which results from electrostatic interaction between compound $\mathbf{1}$ and the dendrimer. Such broadening in IR in the region of $\mathrm{NH}$ and $\mathrm{NH}_{2}$ due to formation of $-\mathrm{NH}_{3}{ }^{+}$is comparable to previously reported encapsulation studies $[5,21]$.

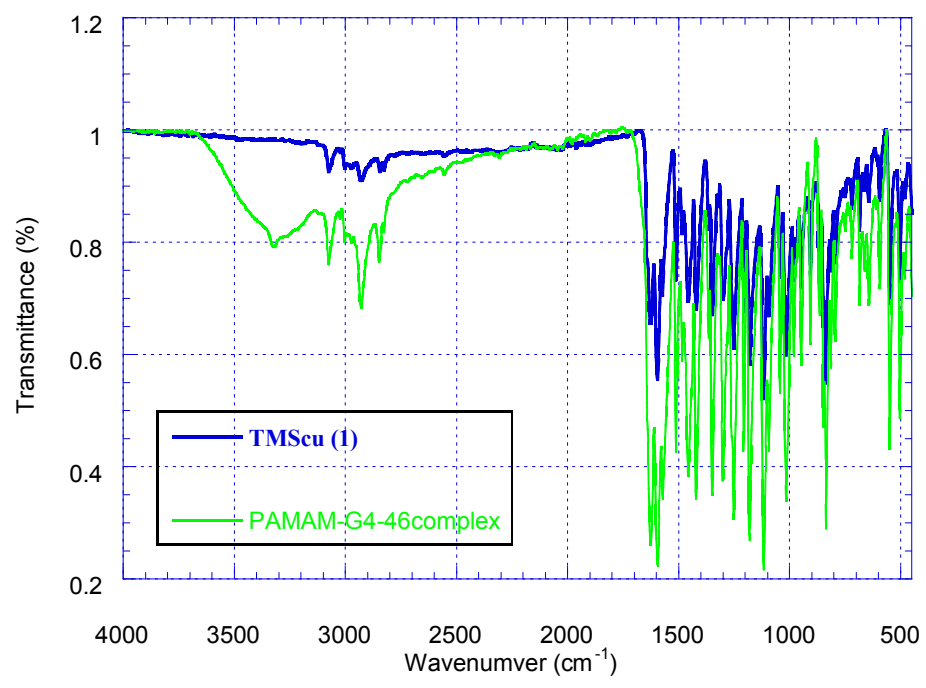

Figure 6. The superimposed IR spectra of TMScu (1) and its dendrimer complex.

The ${ }^{1} \mathrm{H}$ NMR spectra of pure compound 1 and its dendrimer complex are presented in Figures 7 and 8 respectively. As indicated in Figure 7, the ${ }^{1} \mathrm{H}$ NMR spectrum of the complex (PAMAM G4 TMScu (1)) contains signals from both the PAMAM G4 and compound $\mathbf{1}$. The appearance of the 
slight shift of some peaks of compound $\mathbf{1}$ in the compound-dendrimer complex as illustrated by stacked ${ }^{1} \mathrm{H}$ NMR spectra (Figure $9 \mathrm{a}, \mathrm{b}$ ) suggests interactions brought about by an external electrostatic interaction between TMScu (1) and PAMAM. The methoxy protons showed significant signal dynamics with approximately $\delta 0.01$ shifts and visually evident drifted signals showing doubling in PAMAM G4-TMScu complex (green, Figure 9b) suggesting interaction probably involving hydrogen bonding between methoxy oxygen of $\mathbf{1}$ and amino groups of the dendrimer G4.

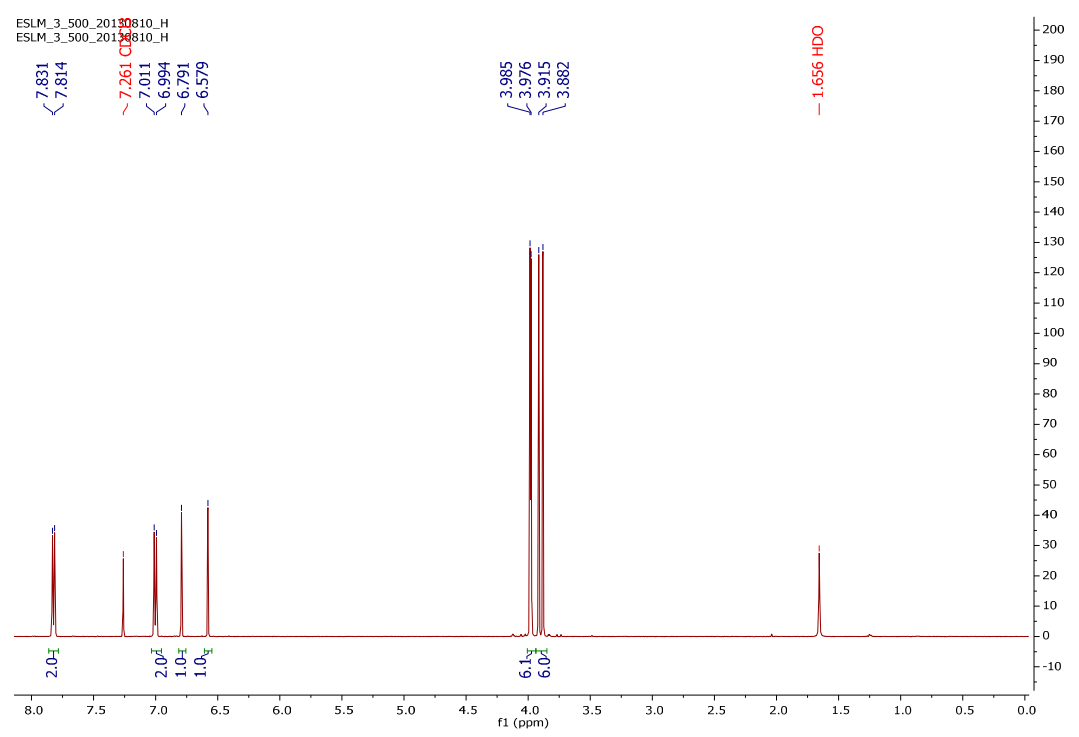

Figure 7. The ${ }^{1} \mathrm{H}$ NMR spectrum of TMScu (1) observed at $499.88 \mathrm{MHz}$ for $\mathrm{CDCl} 3$ solution at $25^{\circ} \mathrm{C}$.

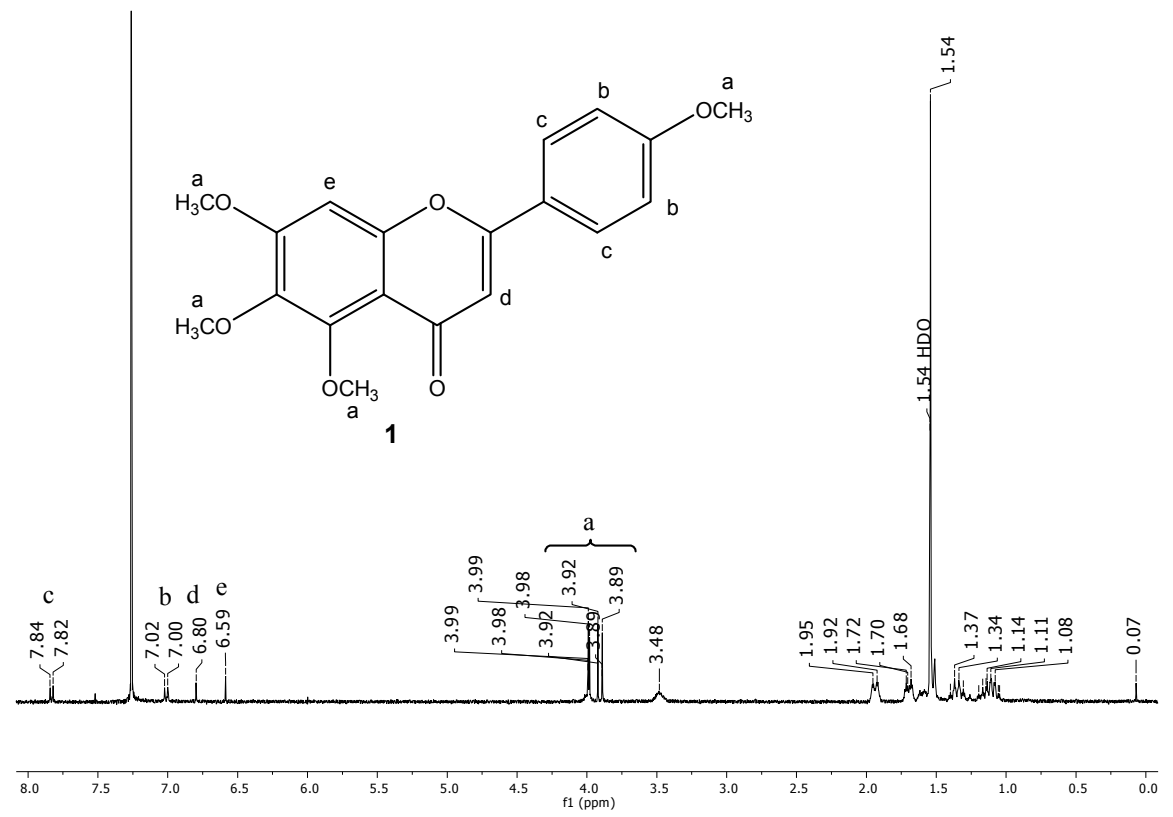

Figure 8. The ${ }^{1} \mathrm{H}$ NMR of spectrum of PAMAM G4-TMScu (1) complex observed at $400 \mathrm{MHz}$ for $\mathrm{CDCl}_{3}$ solution at $25^{\circ} \mathrm{C}$. 


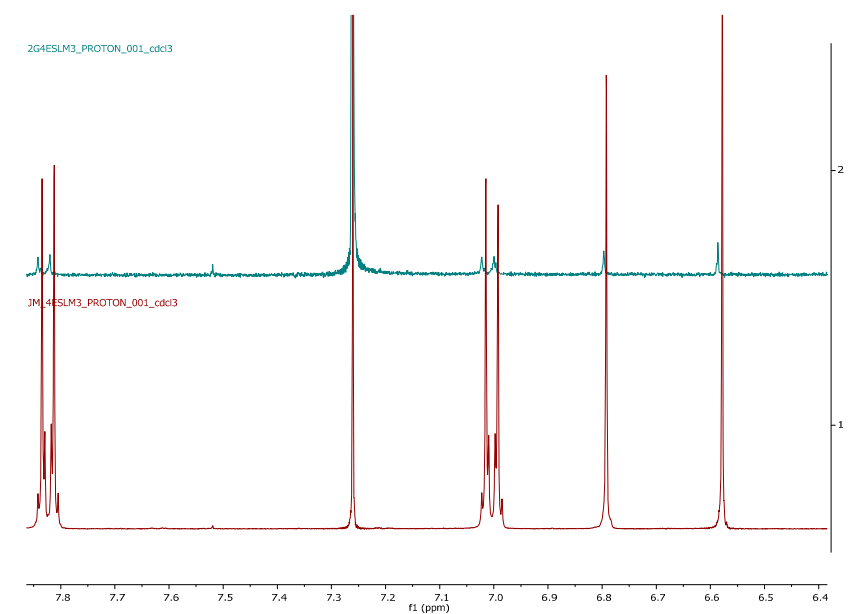

(a)

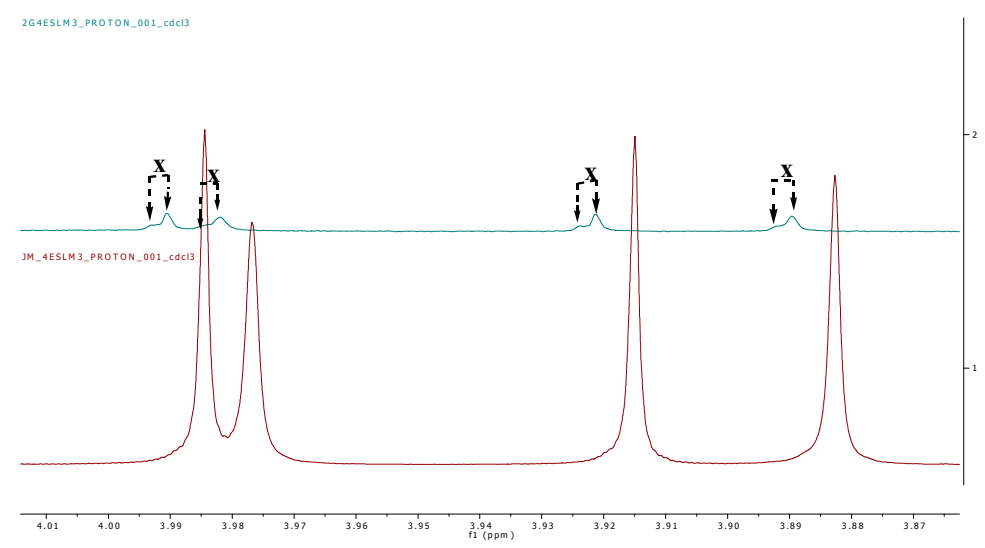

(b)

Figure 9. (a) An expansion of the ${ }^{1} \mathrm{H}$ NMR of spectrum of TMScu (1) encapsulated in PAMAM G4 complex (green) stacked with the ${ }^{1} \mathrm{H}$ NMR of spectrum of TMScu (1) without PAMAM G4 dendrimer observed at $400 \mathrm{MHz}$ for $\mathrm{CDCl} 3$ solution at $25^{\circ} \mathrm{C}$; (b) An expansion of the ${ }^{1} \mathrm{H}$ NMR of spectrum of TMScu (1) encapsulated in PAMAM G4 complex (green) stacked with the ${ }^{1} \mathrm{H}$ NMR of spectrum of TMScu (1) without PAMAM G4 dendrimer observed at $400 \mathrm{MHz}$ for $\mathrm{CDCl} 3$ solution at $25{ }^{\circ} \mathrm{C}$ $(\mathbf{x}=$ drifted signals illustration).

\subsection{Encapsulation Efficiency (EE) and Loading Capacity (LC)}

Encapsulation efficiency (EE) of compound $1\left(\mathrm{C}_{19} \mathrm{H}_{18} \mathrm{O}_{6}, \mathrm{M}=342.3 \mathrm{~g} / \mathrm{mol}\right)$ was $77.8 \% \pm 0.69 \%$, while its loading capacity (LC) was $6.2 \% \pm 0.06 \%$. The encapsulation efficiency is influenced by among other factors the nature of the polymer nanoparticle and the encapsulated drug molecules, polymer-drug ratio and the concentration [1,22]. The value obtained for encapsulation efficiency and loading capacity could be attributed to the intrinsic features of both PAMAM G4 dendrimer and TMScu (1) enabling intermolecular interactions. Furthermore, dendrimers with high molecular weight such as PAMAM G4 would have high loading capacity and encapsulation capacity as opposed to those with lower molecular weight [22]. The phenomenon is also explained by the results of the present study, the EDTA core G4 dendrimer has high molecular weight as well as larger core size and thus has higher loading capacity as observed.

\subsection{Phase Solubility Studies of the TMScu (1)-Dendrimer G4 Formulation}

Measurements of solubilization ability of PAMAM dendrimer TMScu (1) was done, as previously described [5]. Phase solubility diagram was obtained by plotting the equilibrium 
concentration of compound 1 against PAMAM dendrimer concentrations (Figure 10). The solubility increased linearly as function of PAMAM dendrimer concentration albeit with some deviations noticeable at higher concentrations of the dendrimer at $\mathrm{pH}$ 7.2. According to the model developed by Higuchi and Connors [23], the linear relationship between TMScu (1) solubility and PAMAM dendrimer concentration indicated an $\mathrm{A}_{\mathrm{L}}$-type phase diagram particularly at $\mathrm{pH}$. The obtained linear diagrams (Figure 9) suggest the formation of water-soluble complex in solution due to electrostatic and hydrogen bonding interactions. Such an $A_{L}$-type phase solubility profile is obtained when the complex is first order with respect to both the dendrimer (ligands) and the drug (substrate) [23,24]. Generally, the solubility of drug-dendrimer complex increases linearly with increasing dendrimer concentration due to electrostatic interactions between the interactive functional groups of the drug and the amino groups of the dendrimer [3,25]. The reported mechanism of interaction could hold the same for compound 1, as it contains the carbonyl and methoxy functionalities in its structure. Thus, the electrostatic interaction between the primary amine of PAMAM G4 and the carboxyl or methoxy groups of the investigated compound could be responsible for its enhanced solubilization. Furthermore, low solubility at $\mathrm{pH} 4.0$ can be attributed to the differences in $\mathrm{p} K_{a}$ of PAMAM dendrimer at different $\mathrm{pH}$ levels. The study by Jun-Jun et al., found that the amino groups of the dendrimer have different $\mathrm{p} K_{a}$ [25]. The surface of PAMAM dendrimer amines has $\mathrm{p} K_{a}$ that ranges from 7 to 9 while that of tertiary amines ranges from 3 to 6 [25]. Hence as for the present study, the difference in $\mathrm{p} K_{a}$ of the PAMAM G4 dendrimer would suggest that dissociation would change with variation of $\mathrm{pH}$ leading to the variation of solubility with $\mathrm{pH}$.

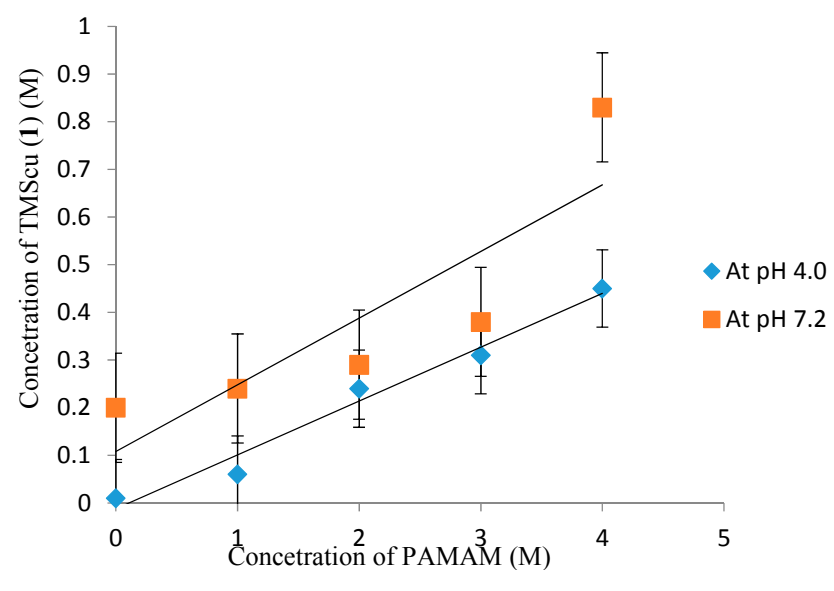

Figure 10. Phase solubility diagram of TMScu (1) in the presence of increasing PAMAM concentration.

\subsection{In Vitro Release of the Loaded TMScu (1)}

In vitro release to investigate the stability and interaction of TMScu-dendrimer complex was done in a phosphate buffered saline (PBS) media at two different $\mathrm{pH}$ values (4.0 and 7.0$)$. In vitro release behavior of loaded compound is presented in Figure 11. For a period of $1 \mathrm{~h}$ at $\mathrm{pH} 4.0$, TMScu (1) showed an initial release of $17 \%$, which later increased up to $36 \%$ for a period of $4 \mathrm{~h}$. The release was nearly maintained for $3 \mathrm{~h}$ (i.e., up to the seventh hour) and then progressively increased. The highest release of $75 \%$ was obtained after $13 \mathrm{~h}$. However, at $\mathrm{pH} 7.0$, the release of TMScu (1) was only $22 \%$ for a period of $13 \mathrm{~h}$. As indicated in Figure 11, the in vitro release of the investigated compound was $\mathrm{pH}$ and ionic interaction dependent. This gives an implication that at $\mathrm{pH} 7$ the TMScu-dendrimer complex formulation was relatively stable as few percentages of the compound were released as compared to that at $\mathrm{pH} 4$. It is generally acknowledged that encapsulation of the compound by amine in basic condition prevents release from the dendrimer [26]. Once the dendrimer enter the cancerous cells or tumor cells which has acidic environment (low $\mathrm{pH}$ ) the amino groups protonate, repel and undergoes conformational change which facilitate the release 
of the compounds [26]. More molecules of TMScu (1) were released at $\mathrm{pH} 4.0$, implying it could also be made available to the targeted tumor cells' physiological environment.

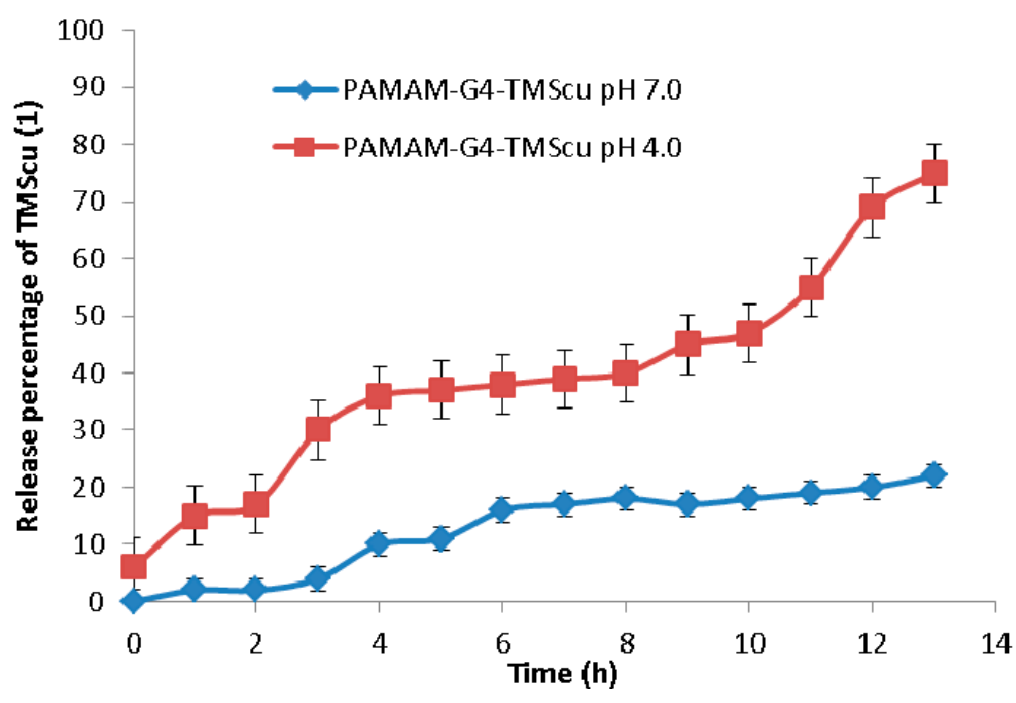

Figure 11. Release profile studies of loaded TMScu (1) from dendrimer-compound complex at $\mathrm{pH} 4$ and 7.

\subsection{Stability Studies of Dendrimer G4-TMScu (1) Formulation}

The study of the physical stability of the formulations at different temperature conditions, that is, 0,27 and $40^{\circ} \mathrm{C}$ to assess the disintegration was carried out and the results presented in Table 1 . For a period of two months, a sign of color changes, turbidity and precipitate formation were observed for formulation stored in light at $40{ }^{\circ} \mathrm{C}$. The formulations/complexes were found to be relatively stable, even at the warmer temperature of $40^{\circ} \mathrm{C}$ when stored under dark conditions, indicating that light had an influence on their stability. The formation of precipitates observed may be attributed to the opening of dendritic structures at higher temperatures [5]. There were no noticeable precipitates, turbidity and color changes for the formulations stored in dark and in light at $27{ }^{\circ} \mathrm{C}$, suggesting that the formulations should be stored in the dark, at low temperature or in a cool place for future applications, should it be found useful.

Table 1. Stability of PAMAM G4-TMScu (1) Formulation.

\begin{tabular}{|c|c|c|c|c|c|c|c|}
\hline \multirow{3}{*}{ Formulation } & \multirow{3}{*}{ Parameter } & \multicolumn{6}{|c|}{ Temperature $\left({ }^{\circ} \mathrm{C}\right)$} \\
\hline & & \multicolumn{3}{|c|}{ Light } & \multicolumn{3}{|c|}{ Dark } \\
\hline & & 0 & 27 & 40 & $\mathbf{0}$ & 27 & 40 \\
\hline \multirow[t]{3}{*}{ PAMAM G4-TMScu (1) } & Color change & - & - & +++ & - & - & + \\
\hline & Turbidity & - & + & + & - & + & ++ \\
\hline & Precipitation & - & + & ++ & - & - & +++ \\
\hline
\end{tabular}

$(-)$ No changes were observed, $(+)$ changes were observed with varied extent.

\section{Experimental Section}

\subsection{Materials and Reagents}

All chemicals and reagents were obtained from commercial sources and used without further purification, unless otherwise stated. Chemicals and reagents included ethylenediaminetetraacetic acid (EDTA) (Rochelle chemicals, Johannesburg, South Africa, (SA), purity: 99.5\%), ethylenediamine (EDA) (M \& B, London, UK, purity: 98.5\%), acetonitrile (Carloerba reagents, Avenue de Valdonne, 
13124 Peypin, France, purity: 99.5\%), ethyl acetate (Fisher chemicals, Loughborough, Leics, UK, purity: 99.98\%), methanol (Fischer chemicals), dicyclohexylcarbodiimide (DCC) (BDH Ltd., London, UK). Trans-2-(3-(4-tert-butylphenyl)-2-methyl-2-propenylidene)malononitrile (DCTB, 99.0\%; Sigma-Aldrich, Stockholm, Sweden) Tetramethylscutellarein (TMScu, 1) was obtained from the Natural Products Research Laboratory, Chemistry Department, University of Dar es Salaam. Solvents used were analytical reagent (AR) grade.

\subsection{General Experimental Procedures}

Reactions were run at room temperature unless stated otherwise. Purification and separation techniques included filtration, evaporation, and centrifugation. Glassware were washed and dried in an oven. The ${ }^{1} \mathrm{H}$ NMR spectra were obtained in $\mathrm{CDCl}_{3}$ using Varian MR 400 and Varian VNMR-S 500 at the Department of Chemistry and Molecular Biology, University of Gothenburg, Sweden. The IR spectra were recorded on Platinum ATR alpha Bruker spectrophotometry and UV-Vis spectroscopy measurements using UV-1800PC Spectophotometer (Shenandoah instruments, Inc., Beijing, China) and were performed at the Chemistry Department, University of Dar es Salaam. The autoflex MALDI-TOF-MS analysis was performed on FlexControl Bruker Daltonics, at Polymer Technolgy Laboratory, Chalmers University of Technology, Gothenburg, Sweden. DCTB was used as the matrix for the MALDI-MS acquisition.

\subsection{Synthesis of Polyamidoamine (PAMAM) Dendrimer Generation 4}

Ethylenediaminetetraacetic acid, EDTA core based PAMAM dendrimers were synthesized by divergent approach with reagent excess using Michael addition and amidation reactions [2]. The Michael addition involved the formation of half dendrimer generations, while in the amidation, two reactions steps were involved: (i) coupling reaction involving activation of carboxylic group and (ii) amidation step, which produced full generation amine terminated G4 PAMAM dendrimer (Scheme 1). In a typical experiment, EDTA core $(6 \mathrm{~g}, 0.0205 \mathrm{~mol})$ in a $250 \mathrm{~mL}$ flask was reacted with ethylenediamine $(48 \mathrm{~mL})$, EDA (2) in the presence of methanol as solvent $(100 \mathrm{~mL})$ and dicyclohexylcarbodiimide (4 g) (DCC, 4) for $48 \mathrm{~h}$ in dark while stirring in a tightly stoppered flask. The product obtained G1, was reacted with methacrylic acid (3) $(30 \mathrm{~mL})$ in the presence of methanol $(100 \mathrm{~mL})$ to give G1.5, and the solvent was evaporated by rotary evaporator. Apart from being a coupling and dehydrating agent, DCC was also used to activate the carboxylic group of EDTA. The dicyclohexylurea (DCU, 5), formed as a byproduct of DCC, was filtered and solvents were evaporated by a rotary evaporator. The activation step reaction was then followed by the amidation reaction (Scheme 2). In this step, the nitrogen lone pair from EDA acted as a nucleophile by reacting with the positive carbonyl carbon from the carboxylic group. These procedures were repeated in several steps to produce the fourth generation dendrimer (PAMAM G4). Synthesized PAMAM G4 was further characterized by IR, UV-Vis, ${ }^{1} \mathrm{H}$ NMR spectroscopy and MALDI-TOF-MS.

\subsection{PAMAM G4}

Yellowish oil: UV ( $\lambda$ max) $250 \mathrm{~nm}$; IR $\left(\mathrm{cm}^{-1}\right) ; 3350.71$ (N-H stretching of primary amine), 3289.27 and 3182.38 (N-H stretching of secondary amine), 2931.22 and 2861.77 (aliphatic and asymmetric $\mathrm{C}-\mathrm{H}$ stretching), 1643.79 (stretching of amide carbonyl) and 1565.49 (C-N stretching in a core). ${ }^{1} \mathrm{H}$ NMR $\left(\mathrm{CDCl}_{3}\right): 4.02 \mathrm{ppm}\left(-\mathrm{CON} \underline{\mathrm{H}}-, \mathrm{H}_{\mathrm{a}}\right) ; 3.48 \mathrm{ppm}\left(-\mathrm{CONHCH}_{2} \mathrm{CH}_{2} \mathrm{NH}_{2}, \mathrm{H}_{\mathrm{b}}\right) ; 1.93 \mathrm{ppm}\left(-\mathrm{C}_{2} \mathrm{CONH}\right.$, $\left.\mathrm{H}_{\mathrm{c}}\right) ; 1.72 \mathrm{ppm}\left(-\mathrm{CONHCH}_{2} \mathrm{CH}_{2}-, \mathrm{H}_{\mathrm{d}}\right) ; 1.69 \mathrm{ppm}\left(\mathrm{NC}_{2} \mathrm{CH}_{2} \mathrm{CONH}-, \mathrm{H}_{\mathrm{e}}\right) ; 1.35 \mathrm{ppm}\left(-\mathrm{NC}_{2} \mathrm{C}_{2} \mathrm{~N}-\right.$, $\mathrm{H}_{\mathrm{f}}$ and $-\mathrm{N}\left(\right.$ tertiary) $\left.-\mathrm{CH}_{2} \mathrm{CH}_{2}, \mathrm{H}_{\mathrm{f}^{\prime}}\right)$.

\subsection{The Autoflex Matrix-Assisted Laser Desorption/Ionization Time of Flight Mass Spectrum (MALDI-TOF-MS) Analysis}

The THF solution of the PAMAM G4 sample and DCTB matrix $(1: 10 v / v)$ was mixed in an Eppendorf tube. Then, $0.5 \mu \mathrm{L}$ of the resulting solution was deposited on two spots on the stainless 
microtiter format MALDI target and dried at room temperature to produce a solid layer [15]. The MALDI-TOF mass spectra of PAMAM G4 dendrimer was acquired by 500 laser shots by autoflex when the instrument was operating in the positive ion linear (flight path $1.22 \mathrm{~m}$ ) mode applying an acceleration voltage of $20.04 \mathrm{kV}$.

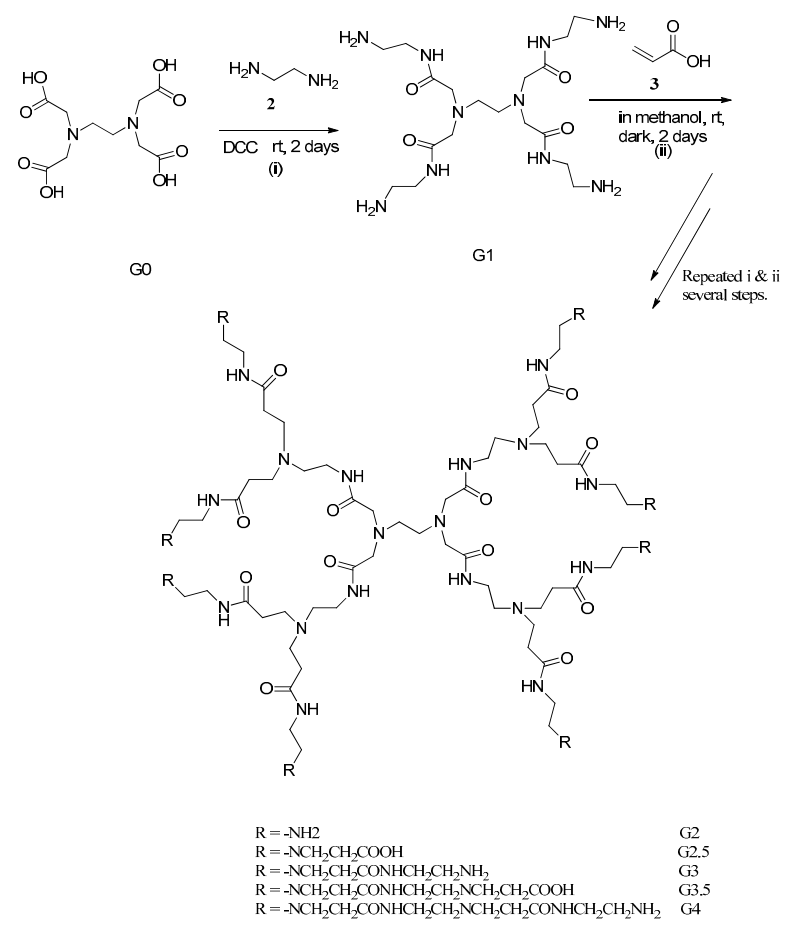

Scheme 1. A divergent synthetic route for PAMAM G4 dendrimer.
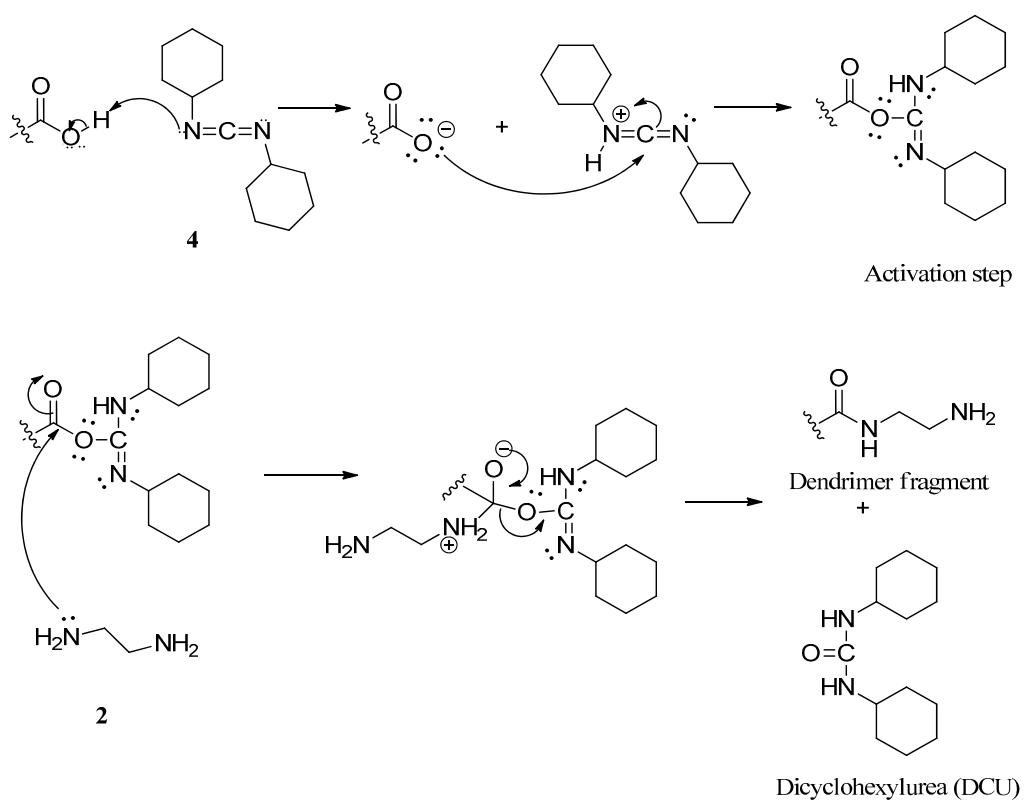

5

Scheme 2. Activation of carboxylic group by Dicyclohexylcarbodiimide (DCC) and amidation step reaction mechanism. 


\subsection{Selection of Model Compound for Encapsulation Study}

The selected model compound (TMScu, 1) with a molecular weight of $342.3\left(\mathrm{~g} \cdot \mathrm{mol}^{-1}\right)$ was a UV active (at $314 \mathrm{~nm}$ ) and sparingly soluble in aqueous media.

\subsection{Encapsulation of TMScu (1) in PAMAM Dendrimer G4}

Encapsulation studies of TMScu, 1 were done according to the reported methods [5,12]. An equal amount of drug to dendrimer ratio in grams was used. At first, $2 \mathrm{mg}$ of compound $\mathbf{1}$ was dissolved in $10 \mathrm{~mL}$ of methanol (i.e., conditions that can dissolve 1), then $2 \mathrm{mg}$ of the dendrimer was added. The reaction was stirred for $30 \mathrm{~h}$ and then the solvent was evaporated under vacuum to remove the methanol completely. This was followed by addition of deionized water to the complex and stirring for another $30 \mathrm{~h}$. Water was filtered and freeze dried to afford a yellow powder of drug-dendrimer complex. Analysis of TMScu (1)-PAMAM-G4 complex was done by IR and NMR spectroscopic techniques to determine if there were absorbance changes or chemical shifts of the functional groups of the compound and/or dendrimer in the complex.

\subsection{Encapsulation Efficiency (EE) and Loading Capacity (LC)}

To determine the encapsulation efficiency (EE) of the encapsulated compound $\mathbf{1}$ in the dendrimer, $2 \mathrm{mg}$ of TMScu (1)-PAMAM complex was mixed with $5 \mathrm{~mL}$ methanol. The solution was then filtered using membrane of $0.22 \mu \mathrm{m}$ pore size to exclude encapsulated from non-encapsulated TMScu (1) and analyzed by UV-Vis spectrophotometer at absorbance of $314 \mathrm{~nm}$ for compound $\mathbf{1}$. The encapsulation efficiency was calculated based on the concentration of the encapsulated compound detected in the formulation over the initial concentration of the compound in methanol as per Equation (1) [1].

$$
\text { Encapsulation efficiency }(\mathrm{EE} \%)=\frac{\left[W_{\mathrm{t}}\right]}{\left[W_{\mathrm{i}}\right]} \times 100 \%
$$

where $W_{\mathrm{t}}$ is the total amount of compound detected in PAMAM formulation and $W_{\mathrm{i}}$ is the total quantity of compound added initially during preparation.

The loading capacity (LC) was also calculated based on Equation (2).

$$
\text { Loading capacity }(\text { LC } \%)=\frac{\text { Weight of TMScu in PAMAM-G4 }}{\text { Weight of PAMAM-G4 }} \times 100 \%
$$

\subsection{Solubilization Study of TMScu (1)}

Phase solubility studies of loaded compound (TMScu, 1) were done according to the previously reported method [5]. The studies were performed in $\mathrm{pH}$ of 4.0 and 7.2 to investigate the effect of $\mathrm{pH}$ on the solubility of the encapsulated compound. The $\mathrm{pH} 4.0$ was chosen to reflect that of cancerous cells, while pH 7.2 is that of blood at normal physiological conditions. In the experiment, an excess of compound $\mathbf{1}$ was added into glass vials containing different concentration of PAMAM dendrimers $(v / v 0.1 \%-0.4 \%)$ in $\mathrm{pH} 7.2$ while in another vial containing only water, excess amount of the compound was added and treated as a control. Both vials were shaken at room temperature for $36 \mathrm{~h}$ in a shaker (Digital Grant Bio Shaker HY-5A, POS 300, USA) and allowed to stand attain equilibrium. The undissolved compound was removed by filtration through a $0.22 \mu \mathrm{m}$ filter paper membrane size and washed twice with distilled water then solubilized in methanol and analyzed by UV-Vis spectrophotometer at the compound's absorbance wavelength. A similar procedure was repeated at $\mathrm{pH} 4.0$ for triplicate experiments and the mean values were used to plot the graphs.

\subsection{In Vitro Release Studies of Loaded Compound (TMScu, 1)}

In vitro release studies of the loaded compound (TMScu, 1) from PAMAM dendrimer-TMScu complex were measured in triplicate in a PBS media at $\mathrm{pH} 7.0$ and $\mathrm{pH} 4.0$. One milligram of 
dendrimer-compound complexes was suspended in $5 \mathrm{~mL}$ of buffer solution ( $\mathrm{pH}=7.0$ and 4.0) in a screw-capped vial. The vials were then placed in a shaker maintained at $37^{\circ} \mathrm{C}$ and shaken horizontally at $240 \mathrm{rpm}$ at a predetermined time interval. The vials were then removed from the shaker and centrifuged for $10 \mathrm{~min}$. The supernatant collected from the vials were then subjected for UV-Vis analyses. The precipitated dendrimer were re-suspended in $5 \mathrm{~mL}$ of fresh buffer solution and then put back into the shaker for continuous in vitro release measurements as per previously described method [21].

\subsection{Stability Studies of TMScu (1)}

The principles of chemical kinetics were employed in the stability studies of the formulation [5]. A stability study was therefore carried out under light and dark conditions with different temperature settings. In the stability study, $1 \mathrm{~mL}$ of formulations was kept in dark (using amber-colored glass vials) and in light (colorless vials) at $0,27^{\circ} \mathrm{C}$ (room temperature) and $40{ }^{\circ} \mathrm{C}$ in a controlled oven for 2 months. Samples were periodically withdrawn and analyzed for any color changes, crystallization, precipitation and turbidity.

\section{Conclusions}

This study investigated the encapsulation and solubilization capacity of the as-synthesized PAMAM dendrimer G4 on a flavonoid tetramethylscutellarein (TMScu, 1) as an approach to enhance the potency of the flavonoid molecules. The synthesis of EDTA core based PAMAM dendrimer of generation four (G4) was designed and successfully achieved via a divergent approach by reacting EDA and acrylic acid in repeated steps. The synthesized G4 dendrimer was characterized by UV-Vis, IR and NMR spectroscopy. The encapsulation of the studied flavonoid (TMScu, 1) into PAMAM G4 dendrimer, solubilization and in vitro release were explored using a combination of IR, UV-Vis and NMR spectroscopic techniques. It was established that amine-terminated dendrimer G4 formed a complex with the flavonoid due to hydrogen bonding and electrostatic interaction as observed in the IR and NMR signal absorption changes. The amine terminated PAMAM G4 enhanced the solubility of the studied compound as a function of dendrimer concentration. Furthermore, $\mathrm{pH}$ was established to have an influence on the solubility with solubility being higher at $\mathrm{pH} 4$ than at $\mathrm{pH}$ 7. The in vitro release studies indicated that PAMAM G4-MScu complex was relatively more stable in neutral medium at $\mathrm{pH} 7$ than at $\mathrm{pH} 4$. Overall, it has been established that PAMAM G4 dendrimer is capable of encapsulating TMScu and hence enhancing its solubility. The dendrimer thus has potential for the bioactivity enhancement of the studied and other related compounds warranting further investigation under in vivo and or in vitro bioassay setups.

Acknowledgments: Authors wish to thank Mate Erdelyi of the Department of Chemistry and Molecular Biology, University of Gothenburg, Sweden for availing access to 1H NMR and MALDI-TOF mass spectra acquisition through existing collaboration with Stephen S. Nyandoro. Daniel M. Shadrack thanks the St. John's University of Tanzania, for MSc fellowship through the Higher Education Loan Board (HESLB) fellowship scheme. Authors thank the University of Dar es Salaam, Chemistry department for providing bench space and analytical equipment.

Author Contributions: Egid B. Mubofu and Stephen S. Nyandoro conceived and designed the experiments. Daniel M. Shadrack performed the experiments. All authors contributed to the preparation of the manuscript.

Conflicts of Interest: The authors declare no conflict of interest.

\section{References}

1. Papadimitrious, S.; Bikiaris, D. Novel self-assembled core-shell nanoparticles based on crystalline amorphous moieties of aliphatic copolyesters for efficient controlled drug release. J. Control. Release 2009, 138, 177-184. [CrossRef] [PubMed]

2. Patel, H.N.; Patel, P.M. Dendrimer applications-A review. Int. J. Pharm. Biol. Sci. 2013, 4, 454-463. 
3. Wong, P.T.; Choi, S.K. Mechanisms and implications of dual-acting methotrexate in follate-targeting nanotherapeutic delivery. Int. J. Mol. Sci. 2015, 16, 1772-1790. [CrossRef] [PubMed]

4. Winnicka, K.; Wroblewska, M.; Wieczorek, P.; Sacha, P.T.; Tryniszewska, E.A. Effect of PAMAM dendrimer on the antibacterial activity of antibiotics with different water solubility. Molecules 2013, 18, 8607-8617. [CrossRef] [PubMed]

5. Prajapati, R.M.; Tekade, R.K.; Gupta, U.; Gajbhiye, V.; Jain, N.K. Dendrimer-mediated solubilisation, formulation development and in vitro-in vivo assessment of Piroxicam. Mol. Pharm. 2009, 6, 940-950. [CrossRef] [PubMed]

6. Vogtle, F.; Richardt, G.; Werner, N. Dendrimer Chemistry: Concepts, Synthesis, Properties, Applications; Wiley-VCH Verlag GmbH \& Co.: KGaA, Weinheim, Germany, 2009.

7. Chahar, M.K.; Sharma, N.; Dobhal, M.P.; Joshi, Y.C. Flavonoids: A versatile source of anticancer drugs. Pharmacogn. Rev. 2011, 5, 1-12. [PubMed]

8. Lin, H.; Zhang, W.; Dong, Z.-X.; Gu, T.; Li, N.-G.; Shi, Z.-H.; Kai, J.; Qu, C.; Shang, G.-X.; Tang, Y.-P.; et al. A new and practical synthetic method for the synthesis of 6-O-methyl-scutellarein: One metabolite of scutellarine in vivo. Int. J. Mol. Sci. 2015, 16, 7587-7594. [CrossRef] [PubMed]

9. Barras, A.; Mezzetti, A.; Richard, A.; Lazzaroni, S.; Roux, S.; Melnyk, P.; Betbeder, D.; Monfilliette-Dupont, N. Formulation and characterization of polyphenol-loaded lipid nanocapsules. Int. J. Pharm. 2009, 379, 270-277. [CrossRef] [PubMed]

10. Siddiqui, I.A.; Adhami, V.M.; Bharali, D.J.; Hafeez, B.B.; Asim, M.; Khwaja, S.I.; Ahmad, N.; Cui, H.; Mousa, S.A.; Mukhtar, H. Introducing nanochemoprevention as a novel approach for cancer control: proof of principle with green polyphenol epiggallocatechin-3-gallate. Cancer Res. 2009, 69, 1712-1716. [CrossRef] [PubMed]

11. Yao, Q.; Hou, S.X.; He, W.L.; Feng, J.L.; Wang, X.C.; Fei, H.X.; Chen, Z.H. Study on the preparation of resveratrol chitosan nanoparticles with free amino groups on the surface. Zhongguo Zhong Yao Za Zhi 2006, 31, 205-208. [PubMed]

12. Pandith, H.; Zhang, X.; Thongpraditchote, S.; Wongkrajang, Y.; Gritsanapan, W.; Baek, S.J. Effect of Siam weed extract and its bioactive component scutellarein tetramethyl ether on anti-inflammatory activity through NF-кB pathway. J. Ethnopharmacol. 2013, 147, 434-441. [CrossRef] [PubMed]

13. Munissi, J.J.E.; Nyandoro, S.S.; Kombo, M.K.; Mgina, A.C.; Gruhonjic, A.; Alao, P.F.; Landberg, G.; Lu, Y.; Bin, W.; Pan, F.; et al. Antimycobacterial and cytotoxic flavonoids from Erythrina schliebenii. Manuscript. J. Nat. Prod. In Preparation to be submitted to, 2015.

14. Hong, H.; Liu, G.Q. Scutellarin attenuates oxidative glutamate toxicity in PC12 cells. Planta Med. 2004, 70, 427-431. [PubMed]

15. Müller, R.; Laschober, C.; Szymanski, W.W.; Allmaier, G. Determination of molecular weight, particle size, and density of high number generation PAMAM dendrimers using MALDI-TOF-MS and nES-GEMMA. Macromolecules 2007, 40, 5599-5605. [CrossRef]

16. Kim, Y.; Klutz, A.M.; Jacobson, K.A. Systemic investigation of polyamidoamine dendrimers surface-modified with poly(ethylene glycol) for drug delivery applications: Synthesis, characterization, and evaluation of cytotoxicity. Bioconjug. Chem. 2008, 19, 1660-1672. [CrossRef] [PubMed]

17. Gautan, S.P.; Sharma, A.K.G.A.; Madhu, T.G. Synthesis and characterization of ester and amine terminated PAMAM dendrimer. Global J. Med. Res. 2013, 13, 6-15.

18. Zheng, P.; Gao, L.; Sun, X.; Mei, S.H. Thermolysis behaviours of the first generation dendritic polyamidoamine. Iran. Polym. J. 2009, 18, 257-264.

19. Garea, S.A.; Ghebaur, A. FT-IR spectroscopy and thermogravimetrical characterization of prodrugs based on different dendritic polymers and antitumoral drugs. Mater. Plast. 2012, 49, 1-4.

20. Ritawidya, R.; Pujiyanto, A.; Mujinali, W.; Setiawan, H.; Ramli, M.; Kurniasih, D.; Yanuar, A.; Mutalib, A.; Kardono, L.B. Synthesis and characterization of poly(amidoamine) dendrimers encapsulated $198 \mathrm{Au}$ nanoparticles. Atom Indones. 2012, 38, 118-126. [CrossRef]

21. Kolhe, P.; Misra, E.; Kannan, R.M.; Kannan, S.; Lai, M. Drug complexation, in vitro release and cellular entry of dendrimers and hyperbranched polymers. Int. J. Pharm. 2003, 259, 143-160. [CrossRef]

22. Intra, J.; Salem, A.K. Fabrication, characterization and in vitro evaluation of poly(D,L-lactide-co-glycolide) microparticles loaded with polyamidoamine-plasmid DNA dendriplexes for applications in non-viral gene delivery. J. Pharm. Sci. 2010, 99, 368-384. [CrossRef] [PubMed] 
23. Higuchi, T.; Connors, K.A. Phase solubility techniques. Adv. Anal. Chem. Instrum. 1965, 4, 117-212.

24. Doile, M.M.; Fortunanto, K.A.; Schmucker, I.C.; Schucko, S.K.; Silva, M.A.S.; Rodrigues, P.O. Physicochemical properties and dissolution studies of dexamethasone acetate- $\beta$-cyclodextrin inclusion complexes produced by different methods. Pharm. Sci. Technol. 2008, 9, 314-321. [CrossRef] [PubMed]

25. Jun-Jun, L.; Zheng-Hong, W.; Qi-Neng, P. The effect of polyamidoamine dendrimers (PAMAM) on the solubility and pharmacokinetics of breviscapine. Acta Pharm. Sin. 2009, 44, 197-202.

26. Kakde, D.; Jain, D.; Shrivastava, V.; Kakde, R.; Patil, A.T. Cancer therapeutics-Opportunities, challenges and advances in drug delivery. J. Appl. Pharm. Sci. 2011, 1, 1-10.

(C) 2015 by the authors; licensee MDPI, Basel, Switzerland. This article is an open access article distributed under the terms and conditions of the Creative Commons by Attribution (CC-BY) license (http://creativecommons.org/licenses/by/4.0/). 